\title{
Endoscopic Transnasal Resection of Trigeminal Schwannoma
}

\author{
Satoshi Kiyofuji ${ }^{1}$ Masahiro Shin ${ }^{1}$ Kenji Kondo ${ }^{2}$ Taichi Kin ${ }^{1}$ Tatsuya Uchida ${ }^{1}$ Nobuhito Saito ${ }^{1}$ \\ ${ }^{1}$ Department of Neurosurgery, The University of Tokyo, Tokyo, Japan \\ ${ }^{2}$ Department of Otolaryngology and Head and Neck Surgery, The \\ University of Tokyo, Tokyo, Japan \\ Address for correspondence Masahiro Shin, MD, PhD, Department of \\ Neurosurgery, The University of Tokyo, 7-3-1 Hongo, Bunkyo-ku, \\ Tokyo 113-8655, Japan (e-mail: shin-nsu@h.u-tokyo.ac.jp).
}

J Neurol Surg B Skull Base 2022;83(suppl S3):e637-e638.

\begin{abstract}
Keywords

- endoscopic transnasal surgery

- gamma knife radiosurgery

- transsphenoid surgery

- trigeminal schwannoma

Trigeminal schwannoma is a rare skull base tumor that can be managed in a variety of treatments including image observation, surgery, stereotactic radiosurgery, such as gamma knife radiosurgery (GKS), and combination of these. Endoscopic transnasal resection is very effective when the tumor is not invading far laterally, or the risk of cerebrospinal fluid (CSF) leak is estimated to be low. A 74-year-old man with a history of prostate cancer and diabetes presented with left oculomotor nerve palsy over a month. Magnetic resonance images (MRI) demonstrated a 25-mm mass in the left cavernous sinus protruding to the left orbit via the superior orbital fissure (-Fig. 1). The patient underwent endoscopic transnasal surgery to decompress the mass. The surgery was uneventful, and postoperative MRI demonstrated satisfactory subtotal resection of the mass (-Fig. 2). The final pathology returned as schwannoma. At 1-year follow-up, the tumor slowly enlarged, and the patient underwent GKS with a marginal dose of $14 \mathrm{~Gy}$. At the last follow-up, 4 months after GKS, the tumor was stable. Unfortunately the patient deceased from the known prostate cancer. Endoscopic transnasal surgery was especially useful in this case, considering the preoperative known cancer state that management of this benign tumor did not ruin the quality of life of this patient while minimizing hospitalization, as achieving satisfactory tumor control with aid from postoperative GKS, minimizing complications.

The link to the video can be found at: https://youtu.be/Q0Ugc2VFV4w.
\end{abstract}

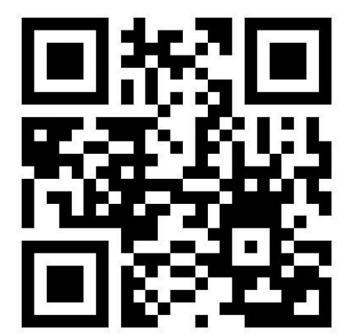

received

September 15, 2020

accepted after revision

December 1, 2020

published online

May 3, 2021

www.thieme.com/skullbasevideos

www.thieme.com/jnlsbvideos

DOI https://doi.org/

10.1055/s-0041-1727122. ISSN 2193-6331.

\footnotetext{
(C) 2021. The Author(s).

This is an open access article published by Thieme under the terms of the Creative Commons Attribution-NonDerivative-NonCommercial-License, permitting copying and reproduction so long as the original work is given appropriate credit. Contents may not be used for commercial purposes, or adapted, remixed, transformed or built upon. (https://creativecommons.org/ licenses/by-nc-nd/4.0/) Georg Thieme Verlag KG, Rüdigerstraße 14, 70469 Stuttgart, Germany
} 


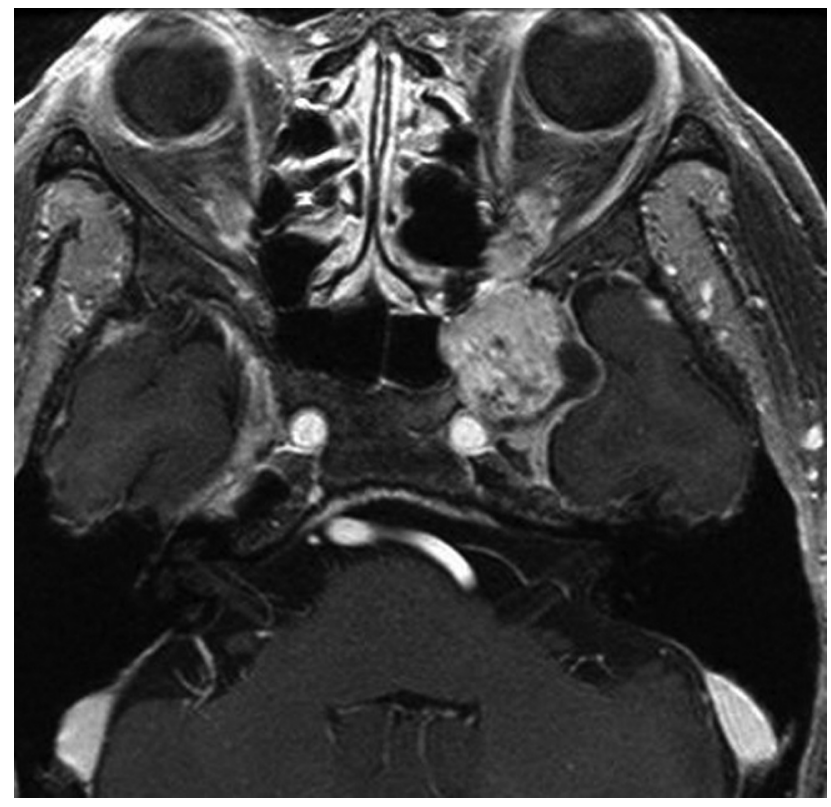

Fig. 1 Preoperative magnetic resonance imaging (MRI) in T1weighted image with contrast enhancement demonstrated a $25-\mathrm{mm}$ mass in the left cavernous sinus protruding to the left orbit via the superior orbital fissure.

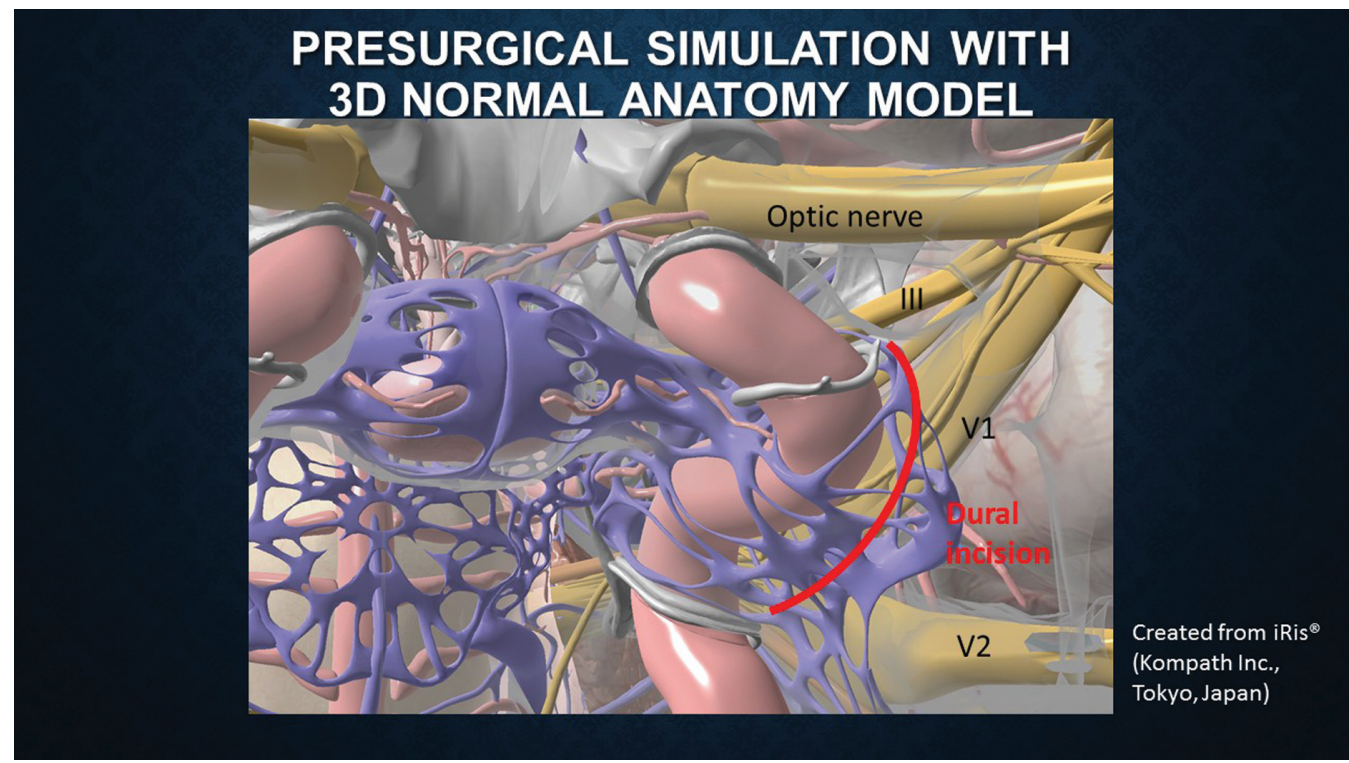

Fig. 2 Presurgical simulation illustration constructed from a three-dimensional computer graphics (3DCG) atlas. Red line demonstrates the dural incision. Image courtesy: iRis (Kompath Inc., Tokyo, Japan).

Conflict of Interest

None declared. 